\title{
ON SIGN ASSIGNMENTS IN LINK FLOER HOMOLOGY
}

\author{
EAMAN EFTEKHARY
}

\begin{abstract}
In this short note, we compare the combinatorial sign assignment of Manolescu, Ozsváth, Szabó and Thurston for grid homology of knots and links in $S^{3}$ with the sign assignment coming from a coherent system of orientations on Whitney disks. Although these constructions produce different signs, a small modification of the convention in either of the two methods results in identical sign assignments, and thus identical chain complexes.
\end{abstract}

\section{INTRODUCTION}

Extending the construction of Heegaard Floer invariants for three-manifolds [OS04b], knot Floer homology was introduced by Ozváth and Szabó [OS04a], and independently by Rasmussen [Ras03], c.f. [Eft05] for the case of homologically non-trivial knots. The construction was refined in OS08] to construct invariants of pointed links, which were defined over $\mathbb{Z} / 2 \mathbb{Z}$. Manolescu, Ozsváth and Sarkar gave a combinatorial description of these link invariants [MOS09, which was further explored by Manolescu, Ozsváth, Szabó and Thurston [MOST07] to present the combinatorially defined grid homology for knots and links in $S^{3}$ with integer coefficients. On the other hand, Alishahi and the author [AE15] refined sutured Floer homology of Juhász [Juh06] (hat version of Heegaard Floer homology for sutured manifolds) to a theory that is defined over integers and includes other versions of Heegaard Floer homology for 3-manifolds and pointed links as special cases. The naturality of the construction is addressed by Alishahi and the author [AE], and independently by Zemke [Zem], but only over $\mathbb{Z} / 2 \mathbb{Z}$.

The goal of this short note is to compare the sign assignment of [MOST07] with the sign assignment of [AE15]. The signs in the construction of [MOST07] are defined combinatorially, by assigning a value in $\{-1,+1\}$ to every empty rectangle which contributes to the differential of the complex. On the other hand, the construction of [AE15] describes how the moduli spaces of holomorphic curves appearing in link Floer homology may be equipped with a coherent system of orientations, denoted by $\mathfrak{o}$. The key is the correct choice of orientation on the moduli spaces of boundary degenerations. In [AE15, $\mathfrak{o}$ is chosen so that the signed count of $J$-holomorphic representatives of every boundary degeneration class with Maslov index 2 is +1 . The signs assigned to holomorphic disks by such $\mathfrak{o}$, which are called coherent systems of orientations with positive boundary degenerations is not compatible with the sign assignment of [MOST07. Alternatively, one may consider coherent systems of orientations with negative boundary degenerations, i.e. those $\mathfrak{o}$ such that the signed count of $J$-holomorphic representatives of every boundary degeneration class with Maslov index 2, is -1 . Then the two conventions for sign assignment agree: Every grid diagram is also a Heegaard diagram and every empty rectangle corresponds to the homotopy class of a Whitney disk. Since the moduli space of holomorphic representatives of every empty rectangle consists of a single point, a coherent system of orientations o gives a map $\mathcal{S}_{\mathfrak{o}}$ : $\operatorname{Rect}^{\circ} \rightarrow\{-1,+1\}$, where Rect $^{\circ}$ denoted the set of all empty rectangles.

Theorem 1.1. For every coherent system of orientations o with negative boundary degenerations on a grid diagram $\mathbb{D}$ for a link $L \subset S^{3}$, the function $\mathcal{S}_{\mathfrak{o}}: \operatorname{Rect}^{\circ} \rightarrow\{-1,+1\}$ is a true sign assignment in the sense of [MOST07, Definition 4.1]. Moreover, every true sign assignment is of the form $\mathcal{S}_{\mathfrak{o}}$ for a uniquely determined coherent system of orientations $\mathfrak{o}$ with negative boundary degenerations. In particular, the grid chain complex associated with $\mathbb{D}$ and the true sign assignment $\mathcal{S}_{\mathfrak{o}}$ is naturally identified with the link Floer complex associated with $(\mathbb{D}, \mathfrak{o})$. 


\section{Proof of THE THEOREM}

Let us assume that $\mathbb{D}$ is a grid diagram, representing some link $L \subset S^{3}$, which does not play a significant role for the purposes of this paper. Then $\mathbb{D}$ consists of a torus $T$, a collection of $n$ horizontal circles $\boldsymbol{\alpha}=\left\{\alpha_{1}, \ldots, \alpha_{n}\right\}$, a collection of $n$ vertical circles $\boldsymbol{\beta}=\left\{\beta_{1}, \ldots, \beta_{n}\right\}$, a collection of $\mathrm{X}$ markings $\mathbb{X}=\left\{\mathrm{X}_{1}, \ldots, \mathrm{X}_{n}\right\}$ and a collection $\mathbb{O}=\left\{\mathrm{O}_{1}, \ldots, \mathrm{O}_{n}\right\}$ of $\mathrm{O}$ markings. We assume that horizontal and vertical circles appear with the (cyclic) order determined by their indices. Let us assume that $L$ has $m$ link components $L_{1}, \ldots, L_{m}$ and that $\mathrm{X}_{i}$ is on $L_{\imath(i)}$. Correspondingly, we obtain a Heegaard diagram

$$
\mathcal{H}=(T, \boldsymbol{\alpha}, \boldsymbol{\beta}, \mathbb{X}, \mathbb{O}) .
$$

The chain complex $\mathrm{CF}(\mathbb{D})$, both in holomorphic curve approach and the combinatorial approach, is freely generated over $\mathbb{Z}\left[\mathfrak{u}_{1}, \ldots, \mathfrak{u}_{n}, \mathfrak{v}_{1}, \ldots, \mathfrak{v}_{m}\right]$ by the grid states. The grid states are in one to one correspondence with permutations $\sigma \in S_{n}$ : associated with every $\sigma$ as above we have a generator $\mathbf{x}_{\sigma}=\left\{x_{1, \sigma(1)}, \ldots, x_{n, \sigma(n)}\right\}$ where $x_{i, j}$ is the unique intersection of $\alpha_{i}$ and $\beta_{j}$. The set of grid states is denoted by $S=S(\mathbb{D})$. For every $\mathbf{x}, \mathbf{y} \in S$, $\operatorname{Rect}^{\circ}(\mathbf{x}, \mathbf{y})$ denotes the set of empty rectangles which connect $\mathbf{x}$ to $\mathbf{y}$. It is clear that there is an inclusion $\operatorname{Rect}^{\circ}(\mathbf{x}, \mathbf{y}) \subset \pi_{2}(\mathbf{x}, \mathbf{y})$. Associated with every $r \in \operatorname{Rect}^{\circ}(\mathbf{x}, \mathbf{y})$, the moduli space $\widehat{\mathcal{M}}(r)=\mathcal{M}(r) / \mathbb{R}$ of $J$-holomorphic representatives of $r$ (divided by the translation action of $\mathbb{R}$ ) consists of a single element. Implicitly, we are of course fixing a path $J$ of almost complex structures on $T$ throughout our discussion.

Let us quickly review the sign assignment coming from the construction of [AE15]. Since all grid states represent the unique $\operatorname{Spin}^{c}$ structure on $S^{3}$, for every $\mathbf{x}, \mathbf{y} \in \mathbf{S}, \pi_{2}(\mathbf{x}, \mathbf{y})$ is non-empty. For every $r \in \pi_{2}(\mathbf{x}, \mathbf{y})$, the determinant line bundle associated with the linearization of timedependent Cauchy-Riemann operator over the space of representative of $r$ is trivial. One may choose a coherent system of orientations as follows. For $\sigma \neq I d_{S_{n}}$, fix the class $r_{\sigma}$ of a Whitney disk in $\pi_{2}\left(\mathbf{x}_{0}, \mathbf{x}_{\sigma}\right)$, where $\mathbf{x}_{0}=\mathbf{x}_{I d_{S_{n}}}$. Moreover, let $a_{1}, \ldots, a_{n} \in \pi_{2}^{\alpha}\left(\mathbf{x}_{0}\right)$ denote the classes of $\alpha$ boundary degenerations, where $a_{i}$ corresponds to the thin cylinder bounded between $\alpha_{i}$ and $\alpha_{i+1}$ (with $\left.\alpha_{n+1}=\alpha_{1}\right)$. Similarly, let $b_{1}, \ldots, b_{n} \in \pi_{2}^{\beta}\left(\mathbf{x}_{0}\right)$ denote the classes of $\beta$ boundary degenerations, where $b_{i}$ corresponds to the thin cylinder bounded between $\beta_{i}$ and $\beta_{i+1}$. The moduli spaces $\mathcal{N}\left(a_{i}\right)$ and $\mathcal{N}\left(b_{j}\right)$ of $J$-holomorphic representatives of $a_{i}$ and $b_{j}$ (respectively) are 2-dimensional, and

$$
\mathrm{G}=\left\{\left(\begin{array}{cc}
a & b \\
0 & \frac{1}{a}
\end{array}\right) \mid a \in \mathbb{R}^{+}, b \in \mathbb{R}\right\}<\operatorname{PSL}_{2}(\mathbb{R})
$$

acts on them. It was observed in [AE15, Section 4] that the orientation on the determinant line bundle may be chosen so that the signed count of points in either of $\widehat{\mathcal{N}}\left(a_{i}\right)=\mathcal{N}\left(a_{i}\right) / \mathrm{G}$ and $\widehat{\mathcal{N}}\left(b_{j}\right)=$ $\mathcal{N}\left(b_{j}\right) / \mathrm{G}$ is +1 . If the aforementioned choice of orientation is fixed over $a_{i}$ and $b_{j}$, the coherent system of orientations is said to have positive boundary degenerations. Alternatively, as we will assume from here on, one may choose the orientation on the determinant line bundle so that the signed count of points in either of $\widehat{\mathcal{N}}\left(a_{i}\right)$ and $\widehat{\mathcal{N}}\left(b_{j}\right)$ is -1 , and the coherent system of orientations is then said to have negative boundary degenerations. Note that each $a_{i} \in \pi_{2}^{\alpha}\left(\mathbf{x}_{0}\right)$ and $b_{j} \in \pi_{2}^{\beta}\left(\mathbf{x}_{0}\right)$ may also be considered as a Whitney disk in $\pi_{2}\left(\mathbf{x}_{0}, \mathbf{x}_{0}\right)$. In both cases, the above choices of orientation is compatible with the equality

$$
a_{1} \star a_{2} \star \cdots \star a_{n}=b_{1} \star b_{2} \star \cdots \star b_{n} \in \pi_{2}\left(\mathbf{x}_{0}, \mathbf{x}_{0}\right) .
$$

Either of the two possible orientations on the determinant line bundle over $r_{\sigma}$ may be chosen by the coherent system $\mathfrak{o}$ of orientations (with negative boundary degenerations). Having fixed the above choices, $\mathfrak{o}$ picks a well-defined orientations on all Whitney disks as follows. Given $r \in \pi_{2}\left(\mathbf{x}_{\sigma}, \mathbf{x}_{\tau}\right)$, we have $r_{\sigma} \star r=p \star r_{\tau}$, where $p \in \pi_{2}\left(\mathbf{x}_{0}, \mathbf{x}_{0}\right)$ is a juxtaposition of the $\alpha$ and $\beta$ boundary degenerations (viewed as classes of Whitney disks in $\pi_{2}\left(\mathbf{x}_{0}, \mathbf{x}_{0}\right)$ ). The orientation on the determinant line bundle over $p$ is determined by the orientation on the determinant line bundles over $a_{i}$ and $b_{j}$. Thus, the orientation on the determinant line bundle over $r$, which is determined 
by the choices of orientation over $p, r_{\sigma}$ and $r_{\tau}$, is uniquely determined by our earlier choices. Associated with each $a_{i}$, one finds an $\alpha$ boundary degeneration class $a_{i}^{\sigma} \in \pi_{2}^{\alpha}\left(\mathbf{x}_{\sigma}\right)$ which is defined by $a_{i} \star r_{\sigma}=r_{\sigma} \star a_{i}^{\sigma}$. Either of the two choices of orientation on the determinant line bundle on $r_{\sigma}$ induces the negative orientation on $a_{i}^{\sigma}$, i.e. the orientation with the property that the number of points in $\widehat{\mathcal{N}}\left(a_{i}^{\sigma}\right)$, counted with sign, is -1 . Similarly, one can define $b_{i}^{\sigma}$, and observe that the number of points in $\widehat{\mathcal{N}}\left(b_{i}^{\sigma}\right)$, counted with sign, is -1 .

Since for every $r \in \operatorname{Rect}^{\circ}, \widehat{\mathcal{M}}(r)$ consists of a single point, the coherent system of orientations $\mathfrak{o}$ may be used to define a map

$$
\mathcal{S}_{\mathfrak{o}}: \operatorname{Rect}^{\circ} \rightarrow\{-1,+1\}
$$

If $\mathfrak{o}^{\prime}$ is another coherent system of orientations, a function $f: S_{n} \rightarrow\{-1,1\}$ describes the difference between $\mathfrak{o}$ and $\mathfrak{o}^{\prime}$, in the sense that

$$
\mathcal{S}_{\mathfrak{o}^{\prime}}(r)=f(\sigma) \cdot f(\tau) \cdot \mathcal{S}_{\mathfrak{o}}(r) \quad \forall r \in \pi_{2}\left(\mathbf{x}_{\sigma}, \mathbf{x}_{\tau}\right) .
$$

By [MOST07, Theorem 4.2], in order to prove Theorem 1.1 it suffices to prove the following statements:

(Sq) For any four distinct $r_{1}, r_{2}, r_{1}^{\prime}, r_{2}^{\prime} \in$ Rect $^{\circ}$ with $r_{1} \star r_{2}=r_{1}^{\prime} \star r_{2}^{\prime}$ we have

$$
\mathcal{S}_{\mathfrak{o}}\left(r_{1}\right) \cdot \mathcal{S}_{\mathfrak{o}}\left(r_{2}\right)=-\mathcal{S}_{\mathfrak{o}}\left(r_{1}^{\prime}\right) \cdot \mathcal{S}_{\mathfrak{o}}\left(r_{2}^{\prime}\right) \text {. }
$$

(V) If $r_{1} \in \operatorname{Rect}^{\circ}\left(\mathbf{x}_{\sigma}, \mathbf{x}_{\tau}\right)$ and $r_{2} \in \operatorname{Rect}^{\circ}\left(\mathbf{x}_{\tau}, \mathbf{x}_{\sigma}\right)$ satisfy $r_{1} \star r_{2}=b_{i}^{\sigma}$, then $\mathcal{S}_{\mathfrak{o}}\left(r_{1}\right) \cdot \mathcal{S}_{\mathfrak{o}}\left(r_{2}\right)=-1$.

(H) If $r_{1} \in \operatorname{Rect}^{\circ}\left(\mathbf{x}_{\sigma}, \mathbf{x}_{\tau}\right)$ and $r_{2} \in \operatorname{Rect}^{\circ}\left(\mathbf{x}_{\tau}, \mathbf{x}_{\sigma}\right)$ satisfy $r_{1} \star r_{2}=a_{i}^{\sigma}$, then $\mathcal{S}_{\mathfrak{o}}\left(r_{1}\right) \cdot \mathcal{S}_{\mathfrak{o}}\left(r_{2}\right)=+1$.

In order to prove $(\mathrm{Sq})$, note that $r=r_{1} \star r_{2}$ has Maslov index 2 , and for generic $J, \widehat{\mathcal{M}}(r)$ is a 1-dimensional manifold. The coherent system of orientations equips $\widehat{\mathcal{M}}(r)$ with an orientation. The boundary of $\widehat{\mathcal{M}}(r)$ correspond to possible degenerations of $r$. Since $r=r_{1} \star r_{2}=r_{1}^{\prime} \star r_{2}^{\prime}$ is decomposed in two ways, one concludes that no boundary degenerations appear as weak limits of $J$-holomorphic curves in $\widehat{\mathcal{M}}(r)$. Moreover, in every degeneration of $r$ to two Whitney disks, either of the Whitney disks is an empty rectangle. Nevertheless, there are at most two such degenerations of $r$. In particular, the boundary of $\widehat{\mathcal{M}}(r)$ is precisely

$$
\left(\widehat{\mathcal{M}}\left(r_{1}\right) \times \widehat{\mathcal{M}}\left(r_{2}\right)\right) \coprod\left(\widehat{\mathcal{M}}\left(r_{1}^{\prime}\right) \times \widehat{\mathcal{M}}\left(r_{2}^{\prime}\right)\right) .
$$

Since the determinant line bundle over $r$ is oriented compatible with the decompositions $r=r_{1} \star r_{2}$ and $r=r_{1}^{\prime} \star r_{2}^{\prime}$, it follows that

$$
\mathcal{S}_{\mathfrak{o}}\left(r_{1}\right) \cdot \mathcal{S}_{\mathfrak{o}}\left(r_{2}\right)+\mathcal{S}_{\mathfrak{o}}\left(r_{1}^{\prime}\right) \cdot \mathcal{S}_{\mathfrak{o}}\left(r_{2}^{\prime}\right)=0
$$

To prove $(\mathrm{V})$, consider the oriented 1-manifold $\widehat{\mathcal{M}}(r)$, where $r=r_{1} \star r_{2} \in \pi_{2}\left(\mathbf{x}_{\sigma}, \mathbf{x}_{\sigma}\right)$. The boundary points of $\widehat{\mathcal{M}}(r)$ are either in correspondence with degenerations of $r$ to two Whitney disks of Maslov index 1, or boundary degenerations. Since $r$ may only be decomposed as $r_{1} \star r_{2}$, the former type of boundary degenerations is in correspondence with $\widehat{\mathcal{M}}\left(r_{1}\right) \times \widehat{\mathcal{M}}\left(r_{2}\right)$. On the other hand, $r$ also corresponds to a $\beta$ boundary degeneration $b_{j}^{\sigma} \in \pi_{2}^{\beta}\left(x_{\sigma}\right)$. Correspondingly, we obtain boundary points which are in correspondence with $\widehat{\mathcal{N}}\left(b_{j}^{\sigma}\right)$. By [AE15, Lemma 5.4], the orientation of $\widehat{\mathcal{N}}\left(b_{j}^{\sigma}\right)$ induced by the coherent system of orientations $\mathfrak{o}$ is the opposite of the orientation induced on it as the boundary of $\widehat{\mathcal{M}}(r)$. Since the signed count of points in $\widehat{\mathcal{N}}\left(b_{j}^{\sigma}\right)$ with the orientation induced by $\mathfrak{o}$ is -1 , it follows that

$$
\mathcal{S}_{\mathfrak{o}}\left(r_{1}\right) \cdot \mathcal{S}_{\mathfrak{o}}\left(r_{2}\right)-(-1)=0 .
$$

The proof of $(\mathrm{H})$ is similar. The only difference is that [AE15, Lemma 5.4] now implies that the orientation of $\widehat{\mathcal{N}}\left(a_{i}^{\sigma}\right)$ induced by $\mathfrak{o}$ agrees with the orientation induced on it as the boundary of $\widehat{\mathcal{M}}\left(r_{1} \star r_{2}\right)$, which gives

$$
\mathcal{S}_{\mathfrak{o}}\left(r_{1}\right) \cdot \mathcal{S}_{\mathfrak{o}}\left(r_{2}\right)+(-1)=0 .
$$


Remark 2.1. Instead of using coherent systems of orientations with negative boundary degenerations, one can modify the convention on the combinatorial side by modifying $(V)$ and $(H)$, so that $\mathcal{S}_{\mathfrak{o}}\left(r_{1}\right) \cdot \mathcal{S}_{\mathfrak{o}}\left(r_{2}\right)=1$ when $r_{1} \star r_{2}=b_{j}^{\sigma}$ and $\mathcal{S}_{\mathfrak{o}}\left(r_{1}\right) \cdot \mathcal{S}_{\mathfrak{o}}\left(r_{2}\right)=-1$ when $r_{1} \star r_{2}=a_{i}^{\sigma}$. These false sign assignments are in one-to-one correspondence with true sign assignments: if $\mathcal{S}_{\mathfrak{o}}$ is a true sign assignment, define

$$
\mathcal{S}_{\mathfrak{o}}^{\prime}(r)=\operatorname{sgn}(\sigma) \cdot \mathcal{S}_{\mathfrak{o}}(r) \quad \forall r \in \operatorname{Rect}^{\circ}\left(\mathbf{x}_{\sigma}, \mathbf{x}_{\tau}\right) .
$$

Then $\mathcal{S}_{\mathfrak{o}}^{\prime}$ is a false sign assignment. False sign assignments may be used in grid homology exactly like true sign assignments, to produce homology groups defined over integers, which are knot/link invariants. This modification has the advantage that boundary degenerations are counted with positive sign, which is perhaps more natural.

\section{REFERENCES}

[AE] Akram Alishahi and Eaman Eftekhary, Tangle Floer homology and cobordisms between tangles, ArXiv:1610.07122.

[AE15] A refinement of sutured Floer homology, J. Symplectic Geom. 13 (2015), no. 3, 609-743.

[Eft05] Eaman Eftekhary, Longitude Floer homology and the Whitehead double, Algebr. Geom. Topol. 5 (2005), 1389-1418.

[Juh06] András Juhász, Holomorphic discs and sutured manifolds, Algebr. Geom. Topol. 6 (2006), 1429-1457.

[MOS09] Ciprian Manolescu, Peter Ozsváth, and Sucharit Sarkar, A combinatorial description of knot Floer homology, Ann. of Math. (2) 169 (2009), no. 2, 633-660.

[MOST07] Ciprian Manolescu, Peter Ozsváth, Zoltán Szabó, and Dylan Thurston, On combinatorial link Floer homology, Geom. Topol. 11 (2007), 2339-2412.

[OS04a] Peter Ozsváth and Zoltán Szabó, Holomorphic disks and knot invariants, Adv. Math. 186 (2004), no. 1, $58-116$.

[OS04b] Holomorphic disks and topological invariants for closed three-manifolds, Ann. of Math. (2) 159 (2004), no. 3, 1027-1158.

[OS08] Holomorphic disks, link invariants and the multi-variable Alexander polynomial, Algebr. Geom. Topol. 8 (2008), no. 2, 615-692.

[Ras03] Jacob Andrew Rasmussen, Floer homology and knot complements, ProQuest LLC, Ann Arbor, MI, 2003, Thesis (Ph.D.)-Harvard University.

[Zem] Ian Zemke, Link cobordisms and functoriality in link Floer homology, arXiv:1610.05207.

School of Mathematics, Institute for Research in Fundamental Sciences (IPM), P. O. Box 193955746, TEHRAN, IRAN

E-mail address: eaman@ipm.ir 